\title{
High Urine Volume and Low Urine Osmolality Are Risk Factors for Faster Progression of Renal Disease
}

\author{
Lee A. Hebert, MD, Tom Greene, PhD, Andrew Levey, MD, Michael E. Falkenhain, MD, and \\ Saulo Klahr, MD
}

\begin{abstract}
- Background: Increased fluid intake slows renal disease progression in animal models. The relevance of these findings to human renal disease is not clear, although increased fluid intake often is recommended to patients with chronic renal insufficiency. This study tested the hypothesis that urine volume, urine osmolality (Uosm), or both are significantly associated with glomerular filtration rate (GFR) decline in patients with chronic renal insufficiency. Methods: This is a retrospective analysis of Modification of Diet in Renal Disease (MDRD) study A patients with (N = 139 ) and without polycystic kidney disease (PKD; $N=442$ ). The key outcome measure was GFR slope in relation to mean 24-hour urine volume and Uosm during follow-up in study $A$ (mean, 2.3 years). Results: The regression of GFR slope on mean follow-up 24-hour urine volume (adjusted for body surface area and MDRD diet and blood pressure group) showed that the greater the urine volume, the faster the GFR decline in patients both with and without PKD. For example, the difference in GFR slope for those with a mean follow-up 24-hour urine volume of 2.4 versus $1.4 \mathrm{~L}$ was $-1.01 \mathrm{~mL} / \mathrm{min} / \mathrm{y}$ (confidence interval, -0.27 to -1.75 ) for patients without $P K D$ and $-1.20 \mathrm{~mL} / \mathrm{min} / \mathrm{y}$ (confidence interval, -0.06 to -2.34 ) for those with PKD. A similar but inverse relationship was shown between GFR decline and mean 24-hour Uosm in patients with $(P=0.01)$ and without PKD $(P=0.001)$. These associations remained significant after adjustment for 13 relevant baseline and follow-up covariates. Conclusion: Sustained high urine volume and low Uosm are independent risk factors for faster GFR decline in patients with chronic renal insufficiency. Thus, high fluid intake does not appear to slow renal disease progression in humans. We suggest that until better evidence becomes available, patients with chronic renal insufficiency should generally let their thirst guide fluid intake. The advice to avoid "pushing fluids" might be particularly important for patients with PKD. Am J Kidney Dis 41:962-971.
\end{abstract}

(C) 2003 by the National Kidney Foundation, Inc.

INDEX WORDS: Urine volume; renal disease progression; polycystic kidney disease (PKD).

$\mathbf{P}$ ATIENTS WITH chronic renal insufficiency commonly are advised to maintain a generous fluid intake. Two recent authoritative publications by nephrologists and for nephrologists recommend "increased" fluid intake in the management of chronic renal disease. ${ }^{1,2}$ The origins of this advice are both historic and contemporary. In the early years of renal physiology, it was shown that urinary urea clearance increased sharply as urine flow rates increased from 1 to 2 $\mathrm{mL} / \mathrm{min} .{ }^{3}$ Lower blood urea nitrogen (BUN) levels from chronic high fluid intake appear to be

From The Ohio State University, Columbus; Cleveland Clinic Foundation, Cleveland, OH; New England Medical Center, Boston, MA; and Washington University, St Louis, MO.

Received September 3, 2002; accepted in revised form January 10, 2003.

Supported in part by the National Institute of Diabetes and Digestive and Kidney Diseases and the Health Care Financing Administration.

No reprints are available.

Address correspondence to Lee A. Hebert, MD, Ohio State University Nephrology, 1654 Upham Dr, Rm N210,

Columbus, OH 43210. E-mail: hebert.1@osu.edu

(C) 2003 by the National Kidney Foundation, Inc.

0272-6386/03/4105-0006\$30.00/0

PII: S0272-6386(03)00193-8 the basis for the historic recommendation to maintain urine volume at $3.0 \mathrm{~L} / \mathrm{d}$ in chronic renal insufficiency. ${ }^{3}$

Contemporary objective evidence of benefit from a high fluid intake in chronic renal insufficiency is provided by studies of experimental ablative nephropathy in rats. ${ }^{4-7}$ Increasing fluid intake to partially decrease the normally high urine osmolality (Uosm) suppressed the maladaptive renal hypertrophy ${ }^{4-6}$ and renal interstitial fibrosis ${ }^{7}$ that are characteristic of that model.

Genetic models of polycystic kidney disease (PKD) in rodents also have been used to indirectly assess the effect of fluid intake on progression of renal disease. In Han:Sprague-Dawley rats, high water intake induced by increased sodium chloride intake increased renal cyst growth. ${ }^{8}$ Conversely, increased sodium bicarbonate intake, which also increased fluid intake, retarded renal cyst growth. ${ }^{9}$ However, in the latter experiments, renal wasting of sodium bicarbonate might have resulted in volume depletion that decreased cyst hydrostatic pressure and growth.

In humans, there has been no prospective study of the effect of fluid intake on renal disease 
progression. However, the Modification of Diet in Renal Disease (MDRD) Study, with its extensive database, provided a unique opportunity to retrospectively determine the association between glomerular filtration rate (GFR) decline and daily urine volume. ${ }^{10}$ Patients with and without PKD were analyzed separately to assess whether an association of fluid intake and renal disease progression (GFR decline) might be different between patients with and without PKD.

\section{METHODS}

The patient population consisted of the study A cohort of the MDRD study (baseline GFR, 25 to $55 \mathrm{~mL} / \mathrm{min} / 1.73 \mathrm{~m}^{2}$ ). Study A was a $2 \times 2$ factorial design in which patients were randomly assigned to two different levels of blood pressure control: the usual goal (mean arterial pressure [MAP] of 102 to $107 \mathrm{~mm} \mathrm{Hg}$ ) or a low goal (MAP $\leq 92 \mathrm{~mm} \mathrm{Hg}$, if tolerated), and two different levels of dietary protein intake: the usual intake $(1.3 \mathrm{~g} / \mathrm{kg}$ of ideal body weight per day) or a low intake $(0.6 \mathrm{~g} / \mathrm{kg}$ of ideal body weight per day). Dietary instructions included avoidance of excess salt intake. Advice on fluid intake was left to the discretion of the physician. GFR decline was estimated from iothalamate clearances performed at 4-month intervals throughout the entire follow-up period. Blood pressure and other clinical testing were performed at 2-month intervals during follow-up. ${ }^{10}$ Patients with $(\mathrm{N}=139)$ and without PKD $(\mathrm{N}=444)$ were analyzed separately. Mean length of follow-up in MDRD study A was 2.3 years. MDRD study B patients (baseline GFR, 13 to $24 \mathrm{~mL} / \mathrm{min} / 1.73 \mathrm{~m}^{2}$ ) were not assessed because the small number of patients with PKD in study B limited the precision of the analyses.

Uosm was calculated as follows:

$\operatorname{Uosm}(\mathrm{mOsm} / \mathrm{L})=[2 \times 24$-hour urine $(\operatorname{sodium}[\mathrm{mEq}]$

$$
+\operatorname{potassium}[\mathrm{mEq}])+35.7
$$

$\times$ 24-hour urine urea nitrogen $(\mathrm{UUN} ; \mathrm{g})]$ /urine volume $(\mathrm{L})$

In the absence of urinary glucose, this method estimates Uosm within $10 \%$ of directly measured Uosm. ${ }^{11}$ Urinary glucose was not measured in the present studies; however, only $3.8 \%$ of MDRD study A patients had diabetes. All had type 2 diabetes.

Plasma osmolality (Posm) was calculated as follows ${ }^{11}$ :

$\operatorname{Posm}(\mathrm{mOsm} / \mathrm{L})=2 \times($ serum sodium $[\mathrm{mEq} / \mathrm{L}]$

$$
+ \text { serum potassium }[\mathrm{mEq} / \mathrm{L}])+\mathrm{BUN}(\mathrm{mg} / \mathrm{dL}) / 2.8
$$

+ blood glucose $(\mathrm{mg} / \mathrm{dL}) / 18$

Baseline daily urine volume and Uosm were calculated as the average of two values: one obtained at entry to baseline, and the second obtained at the end of the 3-month baseline period just before randomization. For each patient, mean follow-up values of daily urine volume and Uosm were computed as the average of monthly measurements of these factors obtained throughout the follow-up period.
To control for differences in body size, all analyses relating GFR decline to urine volume or Uosm were performed using regression models that included body surface area (BSA) as a covariate. Separate regression analyses were used to relate GFR decline to baseline values and mean follow-up values of daily urine volume and Uosm. Daily urine volume was log transformed in the regression analyses because of skewness.

To assess the influence of potential confounding factors in the association of GFR decline with daily urine volume and Uosm, each of these regression analyses was conducted after controlling for each of the following sets of covariates: (1) BSA and randomized treatment group; (2) factors in (1) plus baseline GFR and five other baseline factors shown to independently predict GFR decline in the MDRD study: race, MAP, 24-hour urine protein-creatinine ratio, serum transferrin level, and serum high-density lipoprotein (HDL) cholesterol level ${ }^{10,11}$; and (3) factors in (1) and (2) plus mean follow-up levels of MAP, protein intake estimated from 24-hour UUN, serum sodium concentration, use of diuretics, use of angiotensin-converting enzyme (ACE) inhibitors, use of $\beta$-blockers, use of calcium channel blockers, and 24-hour urine protein-creatinine ratio.

Regression analyses of GFR decline were conducted using two-slope models in which each patient was assumed to have an initial rate of GFR decline in the first 4 months and a possibly different slope thereafter. ${ }^{10}$ The association of baseline and follow-up factors with GFR decline was evaluated as a time-weighted average of regression coefficients relating these factors to GFR decline in the first 4 months and in the subsequent follow-up period. A mixed-effects model was used for these regressions to account for correlations among multiple GFR measurements for the same patients. ${ }^{12}$ Similar analyses were used to relate GFR decline to the use of diuretics, ACE inhibitors, $\beta$-blockers, and calcium channel blockers; mean follow-up Posm levels; and Uosm times daily urine volume (osmolar excretion). For graphic presentation, mixed-effects models were used to obtain mean GFR slopes for patients within quartiles of daily urine volume and Uosm after controlling for baseline BSA and randomized treatment group.

Simple linear regression analyses were used to relate mean follow-up values of log daily urine volume and Uosm to mean follow-up MAP without covariate adjustment. Logistic regression was used to relate the percentage of patients administered diuretics, ACE inhibitors, $\beta$-blockers, or calcium channel blockers at more than $50 \%$ of follow-up visits to mean follow-up values of $\log$ daily urine volume and Uosm. All regression analyses were conducted separately for patients with and without PKD. All mean values are shown as \pm 1 SEM. $P$ of 0.05 or less is considered statistically significant.

\section{RESULTS}

Table 1 lists baseline characteristics of patients in study A with and without PKD. Patients with PKD comprised approximately $25 \%$ of the cohort. Patients with PKD are assumed to have autosomal dominant PKD. 
Table 1. Baseline Demographics of the Study A MDRD Cohort

\begin{tabular}{lr}
\hline \multicolumn{1}{c}{ Renal Diagnosis } & No. of Patients (\%) \\
\hline PKD* $^{*}$ & $141(24.1)$ \\
Glomerular diseases & $141(24.1)$ \\
Hypertensive nephrosclerosis & $32(5.5)$ \\
Tubulointerstitial diseases & $28(4.8)$ \\
Urinary tract diseases & $22(3.8)$ \\
Absence of 1 kidney & $19(3.2)$ \\
Diabetic nephropathy & $17(2.9)$ \\
Hereditary nephritis & $11(1.9)$ \\
Unknown or other & $174(29.7)$ \\
Sex & $357(61)$ \\
$\quad$ Men & $228(39)$ \\
$\quad$ Women & \\
Race & $495(84.6)$ \\
$\quad$ White & $53(9.1)$ \\
$\quad$ Black & \\
History of hypertension & $499(85.3)$ \\
$\quad$ Hypertensive & $86(14.7)$ \\
$\quad$ Nonhypertensive & \\
Age (y) & $325(55.6)$ \\
$\quad<55$ & $260(44.4)$ \\
$\quad \geq 55$ &
\end{tabular}

*Two patients with PKD were excluded from the present analysis because follow-up in the MDRD study was less than 1 year.

Figures 1 and 2 show 24-hour urine volume and Uosm in patients with and without PKD assigned to the usual or low-protein diets during follow-up in the MDRD study, respectively. As shown, the low-protein diet resulted in slightly lower daily urine volumes in patients with and without PKD, probably because urine volume is affected by solute excretion in chronic renal failure. ${ }^{13}$ Note that mean follow-up 24-hour urine volume and Uosm were similar between patients with and without PKD assigned to the same dietary group. To account for differences in urine volume and Uosm associated with the diet group, analyses of GFR slope on urine volume and Uosm were adjusted for effects of the diet group.

Figure 3 shows the regression of GFR slope on mean 24-hour urine volume during follow-up in the MDRD study in patients with and without PKD. This analysis shows that the higher the mean 24-hour urine volume during follow-up, the greater the rate of GFR decline during followup. These associations are significant for patients with and without PKD.

Figure 4 shows the regression of GFR slope on mean 24-hour Uosm during follow-up in the MDRD study in patients with and without PKD. This analysis shows that, in general, the lower the Uosm, the more rapid the GFR decline. This relationship was expected because study A follow-up mean 24-hour urine volume and Uosm correlated significantly and inversely $(r=-0.57$; $P=0.0001$ ).

To assess whether associations shown in Figs 3 and 4 could be explained by association of high daily urine volume and low Uosm with other risk factors for GFR decline, regressions shown in

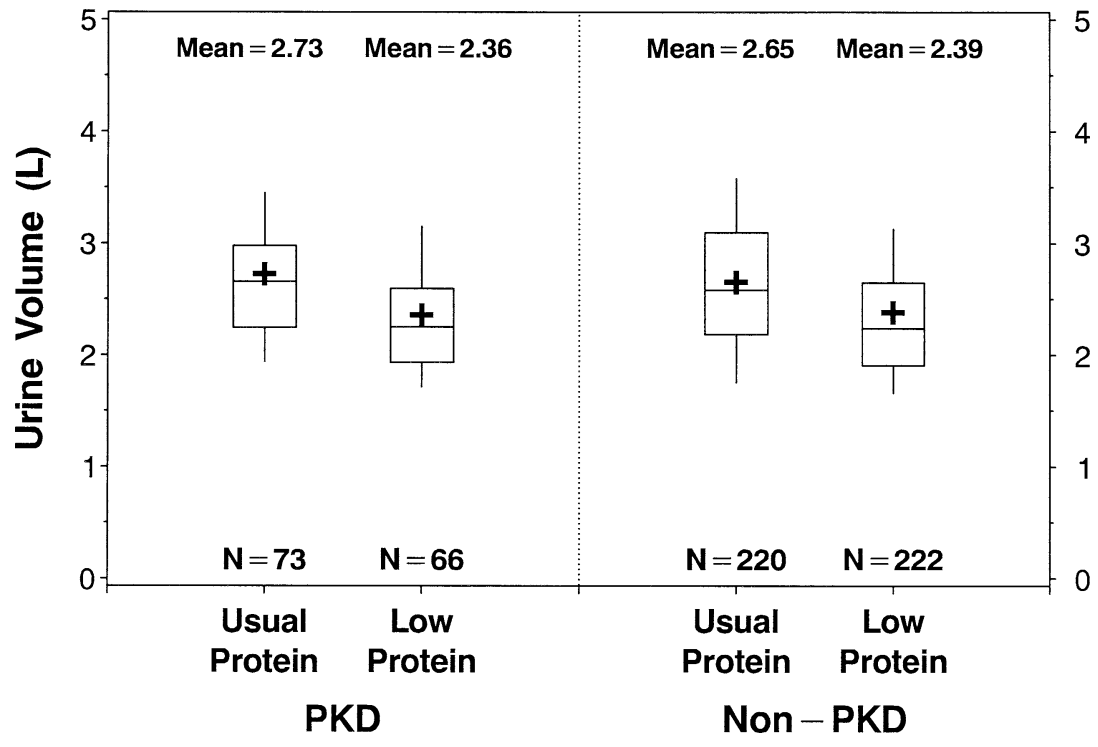

Fig 1. Urine volume in patients with and without PKD according to MDRD dietary group. Note that the lowprotein diet was associated with slightly lower urine volumes. Box plots show 10th, 25th, 50th, 75th, and 90th percentiles. + indicates mean. 
Fig 2. Uosm in patients with and without PKD according to MDRD dietary group. Note that the lowprotein diet was associated with slightly lower urine volumes. Box plots show 10th, 25th, 50th, 75th, and 90th percentiles. + indicates mean.

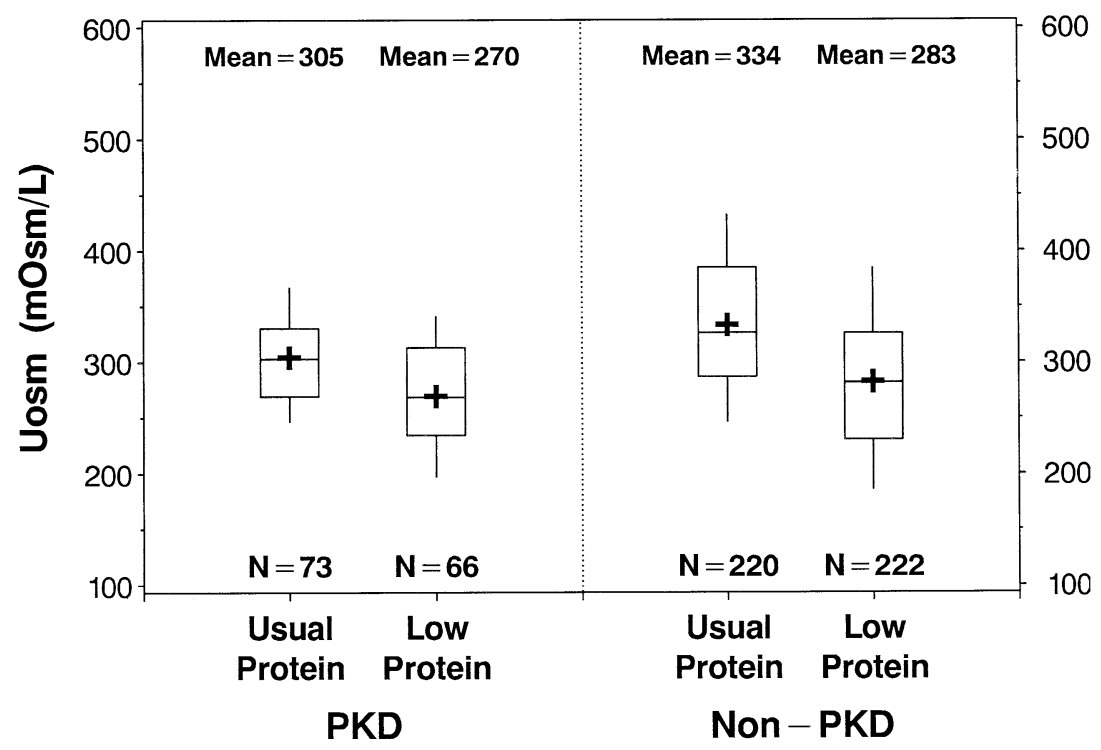

Figs 3 and 4 were adjusted for the baseline covariates GFR, BSA, race, urine protein-creatinine ratio, serum transferrin level, and serum HDL cholesterol level and the follow-up covariates of MAP, protein intake calculated from UUN, serum sodium level, and use of diuretics, ACE inhibitors, $\beta$-blockers, and calcium channel blockers. Results of these analyses are listed in Tables 2 and 3, in which associations of GFR decline with urine volume and Uosm are expressed as regression coefficients: GFR decline (milliliters per minute per year) per 1-L difference in mean follow-up urine volume (Table 2) or GFR decline per $100-\mathrm{mOsm} / \mathrm{L}$ difference in mean follow-up Uosm (Table 3).

As shown in the first data column of Table 2, at baseline the magnitude of the association of GFR decline with daily urine volume is relatively small and generally not significant. Conversely, during follow-up, the magnitude of the association of GFR decline with urine volume is relatively large and highly significant when adjusted only for BSA and diet group (large BSA and usual protein diet are associated with higher

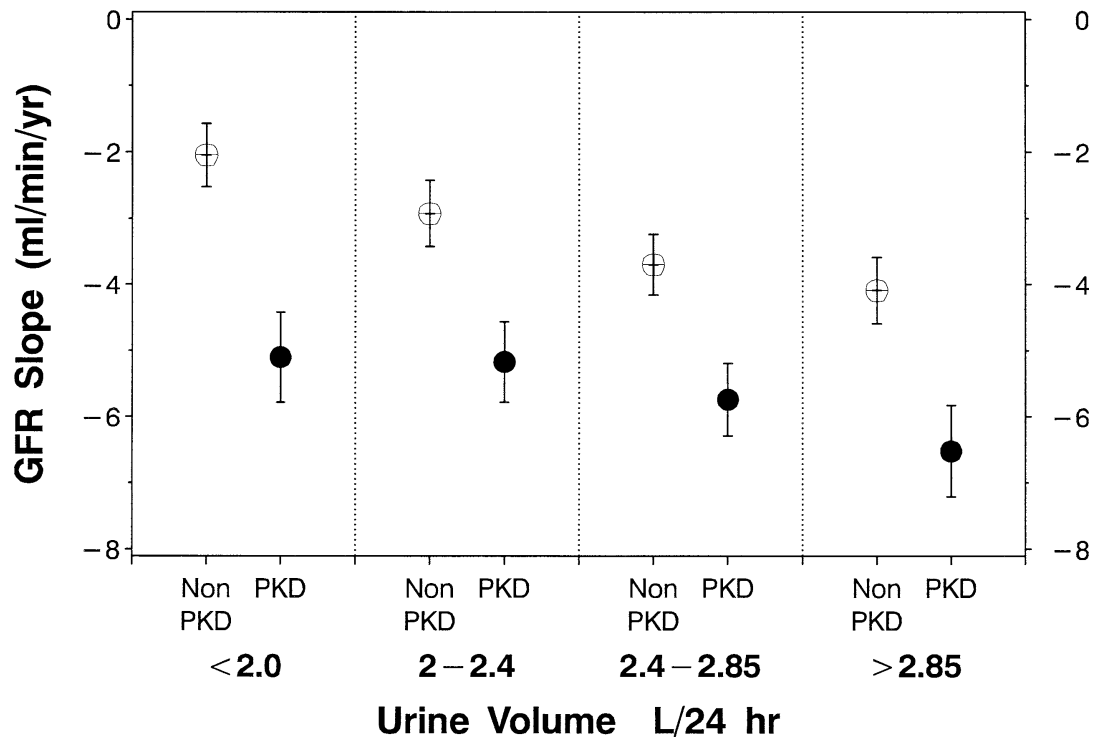

Fig 3. Relationship between GFR slope and mean 24-hour urine volume (arranged in quartiles) in patients without (open symbols) and with PKD (closed symbols) during follow-up in the MDRD study (mean follow-up, 2.3 years). GFR slope was significantly associated with urine volume. The higher the urine volume, the greater the GFR decline in patients with $(P=0.04)$ and without PKD $(P=0.007)$. Means are adjusted for baseline BSA and randomized treatment group. $P$ are based on log urine volume because of positive skewness of values for 24-hour urine volume. 


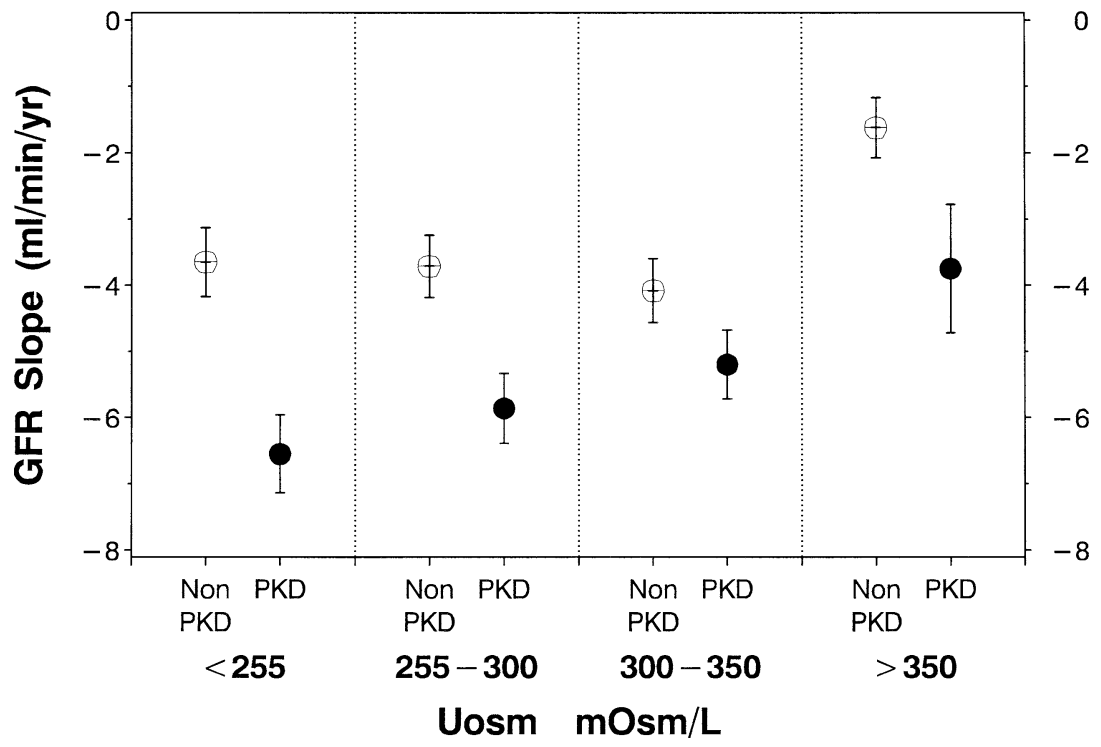

Fig 4. Relationship between GFR slope and mean follow-up Uosm (mOsm/L; arranged in quartiles) in patients with and without PKD in the MDRD study. Conventions used are the same as for Fig 3. Mean GFR slope was significantly associated with mean $\mathrm{mOsm} / \mathrm{L}$. In general, the lower the Uosm, the more rapid the GFR decline in patients with $(P=0.01)$ and without PKD $(P<0.001)$. Mean values are adjusted for BSA and randomized treatment group. urine volume). The stronger statistical association of GFR decline with follow-up daily urine volume compared with baseline daily urine volume may be explained by more precise ascertainment of follow-up values because they are the mean of 12 to 60 measurements/patient. Baseline values are the mean of only 2 measurements/ patient.

As shown in the second and third data columns in Table 2, in patients without PKD, adjust- ment of the follow-up regression coefficient for baseline and follow-up covariates resulted in approximately a $50 \%$ reduction in the magnitude of association of GFR decline with follow-up urine volume, and statistical significance was lost for the association with urine volume with GFR decline. In patients with PKD, adjustment of follow-up regression coefficients for baseline and follow-up covariates had little effect on the magnitude of association of GFR decline with

Table 2. Regression of GFR Slope on Urine Volume: Effect of Adjustment for Baseline and Follow-up Covariates

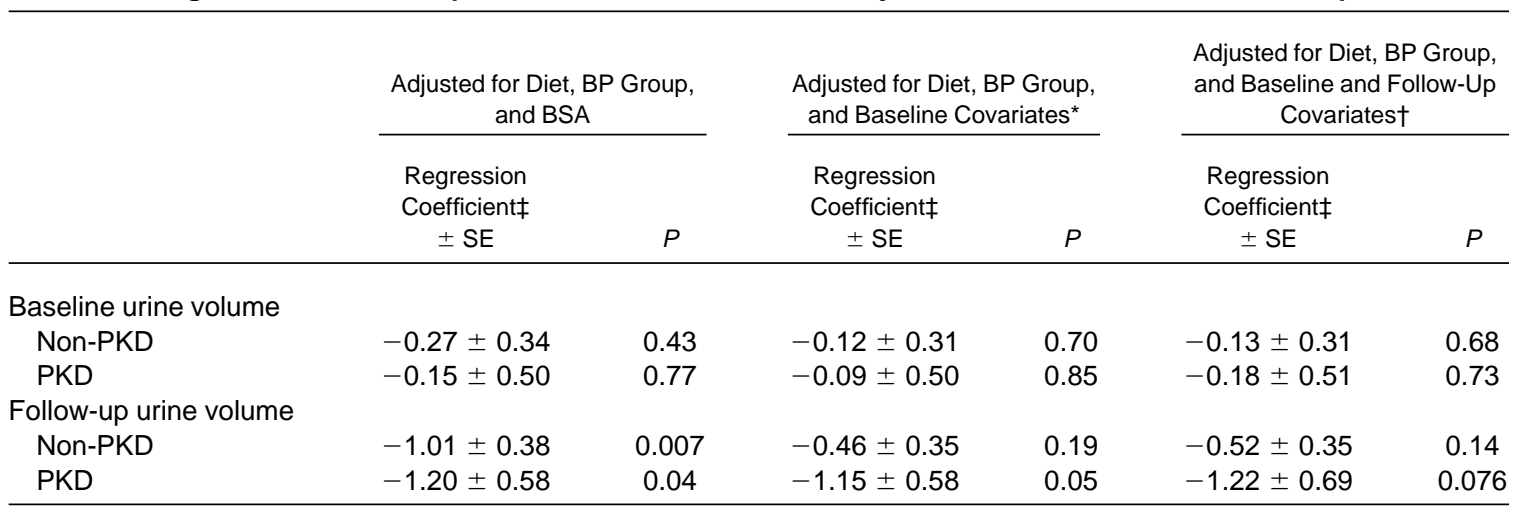

Abbreviation: BP, blood pressure.

*Baseline covariates: GFR, BSA, race, MAP, urine protein-creatinine ratio, serum transferrin level, and serum HDL cholesterol level.

†Follow-up covariates: MAP, protein intake calculated from UUN, serum sodium level, and use of diuretics, ACE inhibitors, $\beta$-blockers, and calcium channel blockers.

¥Regression conducted on log-transformed urine volume because of positive skewness. Regression coefficients expressed as milliliters per minute per year of GFR slope per $1 \mathrm{~L}$ of urine volume when urine volume is equal to follow-up median of $2.4 \mathrm{~L}$. 
Table 3. Regression of GFR Slope on Uosm: Effect of Adjustment for Baseline and Follow-Up Covariates

\begin{tabular}{|c|c|c|c|c|c|c|}
\hline & \multicolumn{2}{|c|}{$\begin{array}{c}\text { Adjusted for Diet, BP Group, } \\
\text { and BSA }\end{array}$} & \multicolumn{2}{|c|}{$\begin{array}{l}\text { Adjusted for Diet, BP Group, } \\
\text { and Baseline Covariates* }\end{array}$} & \multicolumn{2}{|c|}{$\begin{array}{c}\text { Adjusted for Diet, BP Group, } \\
\text { and Baseline and Follow-Up } \\
\text { Covariates } \dagger\end{array}$} \\
\hline & $\begin{array}{l}\text { Regression } \\
\text { Coefficient‡ } \\
\quad \pm \text { SE }\end{array}$ & $P$ & $\begin{array}{l}\text { Regression } \\
\text { Coefficientł } \\
\quad \pm S E\end{array}$ & $P$ & $\begin{array}{l}\text { Regression } \\
\text { Coefficient‡ } \\
\quad \pm \text { SE }\end{array}$ & $P$ \\
\hline \multicolumn{7}{|c|}{ Baseline Uosm } \\
\hline Non-PKD & $0.62 \pm 0.29$ & 0.03 & $0.25 \pm 0.26$ & 0.35 & $0.27 \pm 0.26$ & 0.30 \\
\hline PKD & $0.07 \pm 0.47$ & 0.87 & $-0.03 \pm 0.47$ & 0.95 & $-0.08 \pm 0.48$ & 0.86 \\
\hline \multicolumn{7}{|c|}{ Follow-up Uosm } \\
\hline Non-PKD & $1.25 \pm 0.32$ & $<0.001$ & $0.63 \pm 0.30$ & 0.03 & $0.65 \pm 0.30$ & 0.03 \\
\hline PKD & $1.55 \pm 0.59$ & 0.01 & $1.40 \pm 0.60$ & 0.02 & $1.41 \pm 0.64$ & 0.03 \\
\hline
\end{tabular}

Abbreviation: BP, blood pressure.

${ }^{*}$ Baseline covariates: GFR, BSA, race, MAP, urine protein-creatinine ratio, serum transferrin level, and serum HDL cholesterol level.

†Follow-up covariates: MAP, protein intake calculated from UUN, serum sodium level, and use of diuretics, ACE inhibitors, $\beta$-blockers, and calcium channel blockers.

‡Regression coefficients expressed as milliliters per minute per year of GFR slope $100 \mathrm{mOsm} / \mathrm{L}$ of Uosm.

follow-up urine volume, but the strength of the statistical association was diminished.

Table 3 regression coefficients are positive values because of the inverse relationship between urine volume and Uosm. As shown in the first data column of Table 3, at baseline, the magnitude of association of GFR decline with Uosm is relatively small and significant only for patients without PKD. However, during followup, the magnitude of the association of GFR decline with Uosm is relatively large and highly significant when adjusted for MDRD diet and blood pressure group and BSA. As shown in the second and third columns of Table 3, adjustment of follow-up regression coefficients for baseline and follow-up covariates resulted in approximately a $50 \%$ reduction in the magnitude of the association of GFR decline with follow-up Uosm. However, in patients with and without PKD, statistical significance was maintained.

The statistical association of GFR decline with Uosm was more robust than that of urine volume. This may be explained by greater precision of Uosm than urine volume determination. That is, 24-hour Uosm is calculated with the volume factor in both the numerator and denominator (see Methods) and therefore is affected less by urine collection errors than 24-hour urine volume measurement.

Proteinuria was the baseline and follow-up covariate that most influenced adjustments of regression coefficients (Tables 2 and 3). The effect of proteinuria may be explained because daily urine volume and urine protein-creatinine ratio correlated both at baseline $(P=0.08)$ and during follow-up ( $P=0.002$; data not shown), and proteinuria is a risk factor for faster GFR decline. ${ }^{11}$ The greater effect of the adjustment for proteinuria in patients without PKD may be explained by the greater baseline 24-hour proteinuria in patients without PKD $(1.14 \pm 0.05 \mathrm{~g})$ compared with that of patients with PKD $(0.29 \pm$ $0.04 \mathrm{~g}$ ).

Although the magnitude of the association of GFR decline with follow-up urine volume and Uosm was greater in patients with than without PKD, these differences did not achieve statistical significance $(P=0.29$ for differences in urine volume association; $P=0.36$ for differences in Uosm association).

We examined whether high daily urine volumes were explained better by excess fluid intake or renal sodium and/or water wasting. This analysis showed that patients with high urine volumes tended to have lower serum sodium concentrations (patients with PKD, $P=0.07$; patients without PKD, $P=0.01$; data not shown). Furthermore, in those with the highest urine volumes, urine was frankly hypotonic to plasma (mean Uosm and Posm for those in the lowest quartile of Uosm were $212 \pm 3$ and $301 \pm 1$ $\mathrm{mOsm} / \mathrm{L}$, respectively; $P<0.0001)$. Thus, the 


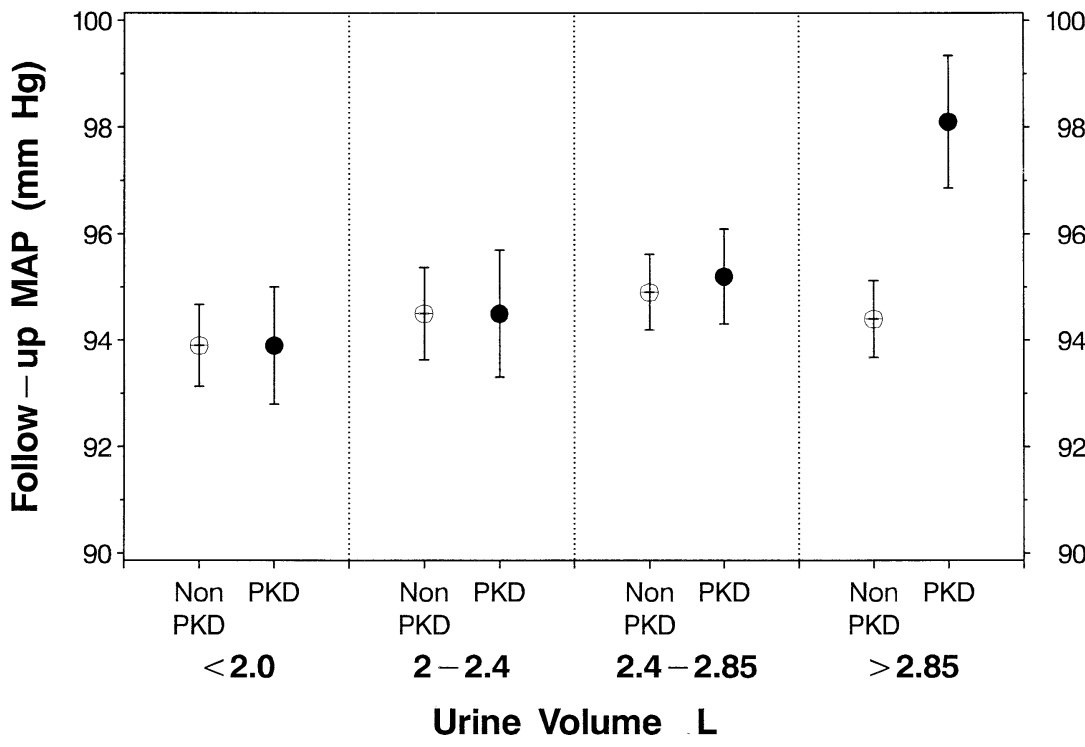

100

98

96

Fig 5. Relationship between follow-up MAP and follow-up mean urine volume in patients without and with PKD of the MDRD study. Conventions used are the same as for Fig 3. In patients without PKD, there was no significant association of MAP with urine volume $(P=$ 0.46). In patients with PKD, there was a significant trend toward higher blood pressure in those with higher urine volume $(P=0.001)$. combination of decreased serum sodium concentration and frankly hypotonic urine suggests that excess water intake was the cause of high daily urine volume, not a renal concentrating defect. Moreover, for patients with the highest daily urine volumes, MAP was either maintained (patients without PKD) or increased (patients with PKD; $P=0.001$; Fig 5). The tendency toward maintained or increased MAP occurred despite a trend for greater diuretic (furosemide) use in those with the highest daily urine volumes (patients with $\mathrm{PKD}, P=0.09$; patients without
PKD, $P=0.002$; Fig 6). Thus, that blood pressure was maintained or increased despite increased diuretic use in those with high urine volumes suggests that primary renal salt wasting also was not the cause of high urine volumes.

Although daily urine volume and diuretic use were significantly associated, diuretic use was not significantly associated with GFR decline $(P=0.28$ for patients without PKD; $P=0.54$ for patients with PKD). For this analysis, diuretic use was a continuous variable (percentage of visits in which diuretics were administered) and
Fig 6. Relationship between diuretic use and mean urine volume during follow-up in patients with and without PKD of the MDRD study. In patients with PKD, there was a nonsignificant trend toward greater diuretic use in those with higher urine volumes $(P=0.09)$. In patients without PKD, there was a significant association between higher urine volume and greater diuretic use $(P=$ 0.002 ). Conventions used are the same as for Fig 3.

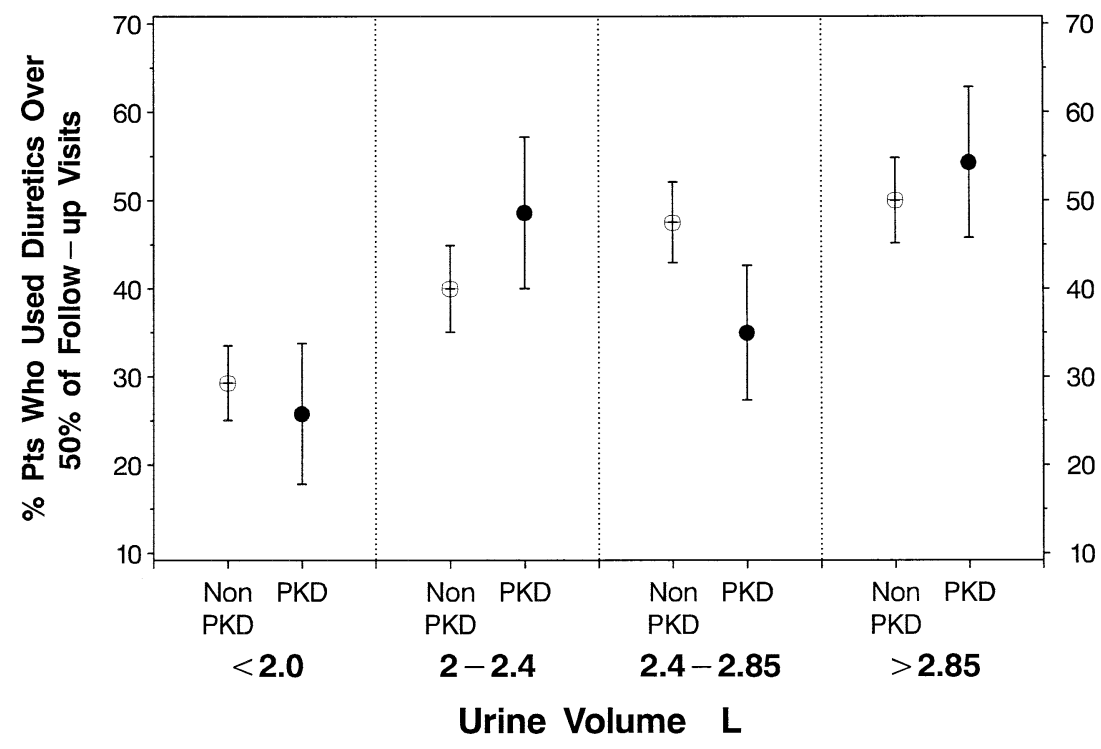


adjustment was made for all baseline and follow-up covariates.

Mean follow-up Posms were $303 \pm 7$ and $302 \pm 6 \mathrm{mOsm} / \mathrm{L}$ in patients with and without PKD, respectively. Follow-up Uosm-Posm ratio in patients with and without PKD was significantly related to GFR decline in a manner similar to that shown for Uosm alone (Table 3). We also examined the association of GFR decline with baseline and follow-up total urinary osmoles (daily urine volume $\times$ Uosm), 24-hour urine sodium level, and 24-hour urine sodium plus potassium levels. None of these associations was significant (data not shown). Thus, the association of GFR decline with urine volume and Uosm was related to rate of urine water excretion, not solute excretion.

Median within-patient coefficient of variation of follow-up daily urine volume was $17.2 \%$ (10th percentile, $11.3 \%$; 90th percentile, $24.7 \%$ ). The between-patient coefficient of variation of the geometric mean of each patient's follow-up daily urine volume values was larger at $30.2 \%$. This indicates that variation between patients in urine volume was larger than variation in urine volume within patients over time. Thus, categorizing patients by geometric mean levels of follow-up daily urine volume is reasonable.

\section{DISCUSSION}

The present study used the MDRD study database to examine retrospectively the relationship between fluid intake (reflected by 24-hour urine volume and Uosm) and renal disease progression (decline in GFR). We tested the hypothesis that fluid intake is significantly associated with GFR decline during follow-up in the MDRD study. Patients with and without PKD were analyzed separately because of evidence from experimental models of renal disease that high fluid intake might increase progression in patients with $\mathrm{PKD},{ }^{8}$ but decrease progression in patients without PKD. ${ }^{4-7}$

We found that for patients with and without PKD, there was a significant association between mean 24-hour urine volume and GFR decline during follow-up in the MDRD study. The higher the mean 24-hour urine volume, the greater the GFR decline. Mean 24-hour urine volume was significantly and inversely related to mean 24hour Uosm. Thus, we also found a significant but inverse association between mean Uosm and GFR decline during follow-up in the MDRD study in patients with and without PKD.

To assess whether the association of faster GFR decline with higher urine volume and lower Uosm could be explained by their association with other risk factors for GFR decline, regressions of GFR decline on urine volume and Uosm were adjusted for relevant baseline and follow-up covariates. In patients without PKD, adjustments resulted in approximately a 50\% reduction in the magnitude of the association of GFR decline with urine volume and Uosm. In patients with PKD, these adjustments had little effect on the magnitude of the association of GFR decline with urine volume and Uosm. This difference between patients with and without PKD was the result of a significant positive correlation between urine volume and urine protein-creatinine ratio. A possible reason for the greater effect of this covariate adjustment in patients without PKD is that they showed greater proteinuria than patients with PKD.

There are two principal hypotheses that can explain the association of high daily urine volume/low Uosm with faster GFR decline. Hypothesis 1 states that high urine volume/low Uosm causes faster renal disease progression. In this scenario, excess fluid intake causes nephron damage (and cyst growth in patients with PKD). Hypothesis 2 states that high urine volume/low Uosm is the result of faster renal disease progression. This scenario can be explained in two ways: (1) faster progression of renal disease directly causes increased urine volume/decreased Uosm by causing greater tubular injury (urinary concentrating defect and/or salt wasting), or (2) faster progression of renal disease indirectly causes increased urine volume by directly increasing thirst.

Our analysis favors hypothesis 1 because patients with and without PKD had findings suggestive of excess water intake. That is, those with the highest daily urine volumes had frankly hypotonic urine, significantly decreased serum sodium concentrating, and maintained or increased blood pressure despite increased diuretic use. If high daily urine volumes had been the result of a urinary concentrating defect and/or salt wasting (hypothesis 2), one or more of the following conditions should have been present in those 
with the highest urine volumes: greater serum sodium concentration, isosthenuria, and lower blood pressure. None of these were present. As discussed, usually the opposite condition was present. Also, although PKD is associated with decreased urine concentrating ability, ${ }^{14} \mathrm{PKD}$ is not known to cause persistent frankly hypotonic urine.

With respect to hypothesis $2 b$ (high urine volume in patients with faster progression of renal disease is the result of a primary increase in thirst), there is no evidence for such a phenomenon in humans with chronic renal insufficiency. The thirst mechanism and antidiuretic hormone $(\mathrm{ADH})$ release have been shown to be normal in patients with chronic renal insufficiency. ${ }^{15}$ Thus, together, our analysis suggests that the association of high urine volume/low Uosm with faster GFR decline can be explained by an adverse effect of excess fluid intake on renal function in patients with chronic renal insufficiency. A hypothesis that could explain how high urine volume might cause faster renal disease progression is that high urine volume increases intratubular volume and pressure, and these stretch forces could induce fibrogenic mechanisms. ${ }^{16,17}$ In patients with PKD, increased intratubular pressure caused by high urine volume also could promote cyst growth. ${ }^{9}$ This effect of urine volume on cyst growth might explain in part the inability of strict blood pressure control, decreased protein intake, ${ }^{18}$ or ACE inhibitors to slow PKD progression. ${ }^{19}$

The present work should not be construed as an endorsement of water restriction in patients with chronic renal insufficiency. This could incur risks. For example, excessively reducing urine volume in patients with PKD could increase the risk for urolithiasis. A recent report ${ }^{20}$ showed that mean 24-hour urine volume in patients with PKD with urolithiasis $(1.75 \mathrm{~L})$ was significantly less than that of patients with PKD without urolithiasis $(2.25 \mathrm{~L})$. Thus, if excess fluid intake is curtailed in patients with PKD, it might be appropriate to use dietary and/or pharmacological measures that reduce the likelihood of urolithiasis. Also, studies in animal models of renal disease showed that water restriction that results in high ADH levels promotes progression of renal disease, in part because ADH induces glomerular hyperfiltration. ${ }^{3-5}$ Thus, in patients with chronic renal insufficiency, water restriction that results in elevated $\mathrm{ADH}$ levels may be as undesirable as excess fluid intake. Bakir and Trinh-Trang$\operatorname{Tan}^{6}$ suggested that the optimum fluid intake to slow progression of experimental renal disease is intake that produces a Uosm-Posm ratio slightly greater than 1.00. Ideally, this would be achieved by reducing excess solute intake and appropriately changing water intake.

In animal studies that showed slowed renal disease progression with increased fluid intake, the animals were able to form hypertonic urine. Thus, it is possible that increased fluid intake might be beneficial in early renal disease when renal concentration is relatively intact. However, we cannot test that hypothesis with MDRD data.

In summary, high fluid intake that results in increased urine volume and low Uosm is not associated with slower renal disease progression. Indeed, high fluid intake might promote progression of renal disease, although this cannot be proved from this retrospective analysis. We suggest the most prudent interpretation of our findings is that until better data become available, patients with chronic renal insufficiency should not be encouraged to ingest a high fluid intake unless it is needed to manage such specific problems as nephrogenic or central diabetes insipidus or urolithiasis. Avoidance of excess fluid intake might be particularly important for those with PKD.

\section{REFERENCES}

1. Kunau RT (ed): Nephrology and Hypertension. Medical Knowledge Self-Assessment Program (ed 2). Philadelphia, PA, American College of Physicians, 1998

2. Stefanski A, Ritz E, Amann K: Factors affecting progression of renal failure-Experimental data, in, Kopple JD, Massry SG (eds): Nutritional Management of Renal Disease Baltimore, MD, Williams \& Wilkins, 1997, pp 277-316

3. Pitts RE: Physiology of the Kidney and Body Fluids (ed 3). Chicago, IL, Year Book Medical Publishers, 1974

4. Bankir L, Bouby N, Trinh-Trang-Tan M-M: Vasopressin-dependent kidney hypertrophy: Role of urinary concentration in protein-induced hypertrophy and in the progression of chronic renal failure. Am J Kidney Dis 17:661-665, 1991

5. Bouby N, Bachmann S, Bichet D, Bankir L: Effect of water intake on the progression of chronic renal failure in the 5/6 nephrectomized rat. Am J Physiol 258:F973-F979, 1990

6. Bakir L, Trinh-Trang-Tan M-M: Urea and the kidney, in Brenner BM (ed): Brenner and Rector's The Kidney (ed 6), chap 15. Philadelphia, PA, Saunders, 2000

7. Sugiura T, Yamauchi A, Kitamura H, et al: High water 
intake ameliorates tubulointerstitial injury in rats with subtotal nephrectomy: Possible role of TGF- $\beta$. Kidney Int 55:18001810, 1999

8. Keith DS, Torres VE, Johnson CM, Holley KE: Effect of sodium chloride, enalapril, and losartan on the development of polycystic kidney disease in Han: SPRD rats. Am J Kidney Dis 24:491-498, 1994

9. Grantham JJ: The etiology, pathogenesis, and treatment of autosomal dominant polycystic kidney disease: Recent advances. Am J Kidney Dis 28:788-803, 1996

10. Klahr S, Levey A, Beck G, et al: The effects of dietary protein restriction and blood pressure control on the progression of chronic renal disease. N Engl J Med 330:877-884, 1994

11. Hunsicker LG, Adler S, Caggiula A, et al: Predictors of the progression of renal disease in the Modification of Diet in Renal Disease study. Kidney Int 51:1908-1919, 1997

12. Laird NM, Ware JH: Random-effects models for longitudinal data. Biometrics 38:963-974, 1982

13. Feinfeld DA, Danovitch GM: Factors affecting urine volume in chronic renal failure. Am J Kidney Dis 10:231235,1987

14. Gabow PA, Kaehny WD, Johnson AM, et al: The clinical utility of renal concentrating capacity in polycystic kidney disease. Kidney Int 35:675-680, 1989
15. Argent NB, Burrell LM, Goodship THJ, Wilkinson R, Baylis PH: Osmoregulation of thirst and vasopressin release in severe chronic renal failure. Kidney Int 39:295-300, 1991

16. Gruden G, Thomas S, Burt D, et al: Interaction of angiotensin II and mechanical stretch on vascular endothelial growth factor production by human mesangial cells. J Am Soc Nephrol 10:730-737, 1999

17. Riser B, Cortes P, Heilig C, et al: Cyclic stretching force selectively up-regulates transforming growth factorbeta isoforms in cultured rat mesangial cells. Am J Pathol 148:1915-1923, 1996

18. Klahr S, Breyer JA, Beck GJ, et al: Dietary protein restriction, blood pressure control, and the progression of polycystic kidney disease. J Am Soc Nephrol 5:2037-2047, 1995

19. Maschio G, Alberti D, Janin G, et al: Effect of the angiotensin-converting-enzyme inhibitor benazepril on the progression of chronic renal insufficiency. The AngiotensinConverting-Enzyme Inhibition in Progressive Renal Insufficiency Study Group. N Engl J Med 334:939-945, 1996

20. Grampsas SA, Chandhoke PS, Fan J, et al: Anatomic and metabolic risk factors for nephrolithiasis in patients with autosomal dominant polycystic kidney disease. Am J Kidney Dis 36:53-57, 2000 